Article

\title{
Green Formation of Spherical and Dendritic Silver Nanostructures under Microwave Irradiation without Reducing Agent
}

\author{
Monir Noroozi, Azmi Zakaria *, Mohd Maarof Moksin, Zaidan Abd Wahab and Alam Abedini \\ Physics Department, Universiti Putra Malaysia, 43400 UPM Serdang, Selangor D.E., Malaysia; \\ E-Mails: monir.noroozi@gmail.com (M.N.); maarof@science.upm.edu.my (M.M.M.); \\ zaidan@science.upm.edu.my (Z.A.W.); abedini_alam@yahoo.com (A.A.) \\ * Author to whom correspondence should be addressed; E-Mail: azmizak@gmail.com; \\ Tel.: +603-89466650; Fax: +603-42705481.
}

Received: 5 April 2012; in revised form: 4 June 2012 / Accepted: 11 June 2012 /

Published: 28 June 2012

\begin{abstract}
The rapid and green formation of spherical and dendritic silver nanostructures based on microwave irradiation time was investigated. Silver nanoparticles were successfully fabricated by reduction of $\mathrm{Ag}^{+}$in a water medium and using polyvinylpyrrolidone (PVP) as the stabilizing agent and without the use of any other reducing agent, and were compared with those synthesized by conventional heating method. UV-vis absorption spectrometry, transmission electron microscopy (TEM), atomic absorption spectroscopy (AAS) and photon correlation spectroscopy (PCS) measurements, indicated that increasing the irradiation time enhanced the concentration of silver nanoparticles and slightly increased the particle size. There was a lack of large silver nanoparticles at a high concentration, but interestingly, the formation and growth of silver dendrite nanostructures appeared. Compared to conventional heating methods, the silver nanoparticle suspension produced by irradiated microwaves was more stable over a six-month period in aqueous solution without any signs of precipitation.
\end{abstract}

Keywords: microwave; silver nanostructures; silver nanoparticles 


\section{Introduction}

The study of nanofluids with metal nanoparticles (NPs), particularly silver nanoparticles (AgNPs), is important in order to be utilized in different fields such as thick-film conductor conductivities, optical biosensors, biomedicine and medicine because of their antibacterial properties [1]. The high quality and nearly monodispersed NPs contribute to the size-dependent nanomedicine applications of AgNPs. In order to get dispersed silver nanoparticles in solvents, polyvinylpyrrolidone (PVP) is one of the commonly used agents that cover the AgNPs surface. The chain length of the surfactant polymer is protective and has been employed as a stabilizer and for the control of morphologies and sizes of $\mathrm{Ag}$ nanostructures [2]. Chemical reduction methods are usually used to synthesize AgNPs. Most chemical reducing reactions require elevated temperatures for a higher reaction rate. The energy used to heat up the media can be conventional heating [3], laser irradiation [4], ultrasound [5], UV irradiation [6], etc.

Generally, microwave (MW) technology is a viable avenue for the greener synthesis of nanomaterials [7-9] and metallic nanoparticles [10-13] because it is clean, rapid, and simple to use. Fast nucleation during the reaction leads to formation of high concentrations and more uniform size distributions [14]. However, MW irradiation should be more environmentally friendly and requires less energy than conventional methods. MW heating conditions allow not only the preparation of spherical nanoparticles within a few minutes but also the formation of dendrites. Depending on the MW irradiation time, it is possible to produce different shapes of Ag nanostructures. Well-defined Ag dendrites have been prepared by reduction of $\mathrm{AgNO}_{3}$ in $\mathrm{N}, \mathrm{N}$-dimethyformamide (DMF) in the presence of PVP under MW irradiation [15]. The synthesis of Ag NP using a number of chemical reducing agents including $\mathrm{NaBH} 4$, ethanol, ethylene glycol and DMF has been well demonstrated by several groups $[3,11,16]$. However, there are a few reports on the use of water solvents in the absence of any reducing agents. Metal colloids in the absence of any reducing agents are free of pollution and can be applied in biomedical system. Besides, the characterization of single dispersed or hydrodynamic agglomerates of nanoparticles is also necessary in order to understand the potential toxicity of NPs on biological systems to ensure that results are reproducible [17]. The rate of silver reduction by water is significantly higher due to its high dielectric values (a dielectric loss of 12 and a dielectric constant of 78). Dielectric heating raises the temperature of the total volume of the reactants by transforming energy selectively in microwave absorbing materials [18]. In particular, the silver nanoparticles can be promptly protected in the water by using a few minutes of MW heating technique.

With higher temperatures, an increasing growth rate of NPs and larger NPs were obtained. In addition, the reduction reaction was completely stopped because the silver reaction could not happen at high temperatures in the presence of water solvent due to its boiling point temperature. Therefore, this corresponds to the agglomeration of silver NPs, and hence controlling the particle growth process is not very easy in this method. However, the reaction rate increased with increasing irradiation time in the microwave system [19]. The removal of high temperatures in microwave heating is possible, since it allows for more reduction reactions of $\mathrm{Ag}$ nanoparticles. Moreover, the MW heating process can produce nanoparticles very quickly and with a very narrow distribution.

In this work, we proposed a green synthesis method by reducing $\mathrm{AgNO}_{3}$ with different $\mathrm{MW}$ reaction times at a moderate temperature for the preparation of silver nanostructures. Water was chosen as the environmentally benign solvent in the preparation. Secondly, PVP as an environmentally 
benign polymer was selected as a ligand as well as nontoxic reducing agents, which have been used as food additives [20]. Finally, microwave irradiation was used as the heating style. The influence of irradiation time on the particle size and distribution of AgNPs was characterized not only by using UV-vis spectra and transmission electron microscopy (TEM) images but also by photon correlation spectroscopy (PCS) which is a novel technique allowing for the simultaneous measurement of particle size and their agglomeration state in suspensions [21]. A conventional heating method was also employed to synthesize AgNPs for a comparison of room temperature with $60^{\circ} \mathrm{C}$.

\section{Experimental Section}

\subsection{Materials and Methods}

Firstly, $\mathrm{AgNO}_{3}\left(99.98 \%, 4.35 \mathrm{~g} / \mathrm{cm}^{3}\right)$ was used as the silver precursor, which was obtained from Merck (Darmstadt, Germany). PVP (K25, MW-29000, Aldrich Chemistry, $1.2 \mathrm{~g} / \mathrm{cm}^{3}$ ) was used as a stabilizer for the fabrication of AgNPs. In a typical procedure, $\mathrm{AgNO}_{3}(0.3 \mathrm{~g})$ and PVP $(0.3 \mathrm{~g})$ were dissolved separately in $25 \mathrm{~mL}$ of water and were stirred for $15 \mathrm{~min}$. Then one was added to the other. The resultant solution was stirred for $10 \mathrm{~min}$ the resulting solution was cooled to room temperature. The corresponding solution was placed into a Pyrex glass cylinder container with a volume of $60 \mathrm{~mL}$. A series of Ag solutions were prepared in a microwave oven (Panasonic, NN-K574MF, multi-mode, rated power $1100 \mathrm{~W}, 2.45 \mathrm{GHz})$ at different irradiation times $(20,40,60$ and $90 \mathrm{~s}(\mathrm{~W} 1-\mathrm{W} 4))$, with a pause every $20 \mathrm{~s}$ to cool the reaction vessel. The temperature of the sample was controlled $\left(\sim 60^{\circ} \mathrm{C}\right)$ to prevent intense boiling of the solvents as well as aggregation of metal NPs. After microwave irradiation, the mixture was allowed to cool to room temperature.

The temperature of a $\mathrm{MW}$ oven can reach $60{ }^{\circ} \mathrm{C}$ in $20 \mathrm{~s}$. For the conventional heating method, a portion of a similar concentration and treatment of silver nitrate and PVP in water was heated on a hot plate from room temperature to $60{ }^{\circ} \mathrm{C}$ during about $20 \mathrm{~min}$. This was done input a similar amount of heat energy as with the MW heating, but at a slower heating rate. After the reaction, the microwave power was turned off and the sample was cooled to room temperature.

\subsection{Characterization Methods}

The fabricated colloidal AgNPs were characterized by UV-vis spectroscopy (UV-1650 PC-Shimadzu), TEM (Hitachi H-7100) for observations of AgNPs in dry condition, aided by UTHSCSA Image Tool (version 3.0; Texas, USA) for software particle size distributions, and PCS (Nanophox Sympatec GmbH) for particle size characterization in aqueous solutions. Samples for PCS analysis were diluted and then placed in a clean vial for measurement, and each experiment had to be repeated four times. The scattered light from a laser beam exhibits a wavelength shift due to Brownian motion of the suspended particles and from it the particle size distribution can be obtained. An atomic absorption spectrometer (AAS-S Series; Thermo Scientific, San Jose, CA, USA) was used to measure the concentration of AgNPs in the solutions. 


\section{Results and Discussion}

\subsection{MW Irradiation}

A microwave oven converts only part of its electrical input into microwave energy. The efficiency was determined by measuring the microwave output power, divided by the power consumption of the oven [22]. The output power absorbed by the sample is different from the nominal power given by the microwave instrument, and normally this effective power is determined by means of a calorimetric method [23]. The experimental results show that the maximum output power of existing microwave ovens is $680 \mathrm{~W}$, which is $61.8 \%$ of the nominal value of $1100 \mathrm{~W}$.

In this work, the color of the prepared samples at different MW-irradiation times at a moderate temperature of $60{ }^{\circ} \mathrm{C}$ gradually changed from colorless for $\mathrm{AgNO}_{3} /$ water suspension (W0) to brown (W1, W2) and finally to dark brown (W3, W4) depending on the absorbation time which indicated the formation of AgNPs in the water suspension. It has been suggested that the color of colloidal Ag depends on the size and the shape of clusters as well as on the surrounding medium [24].

\subsubsection{TEM Images}

Figure 1 shows TEM images and their resultant size distributions of AgNPs in the colloidal solutions under MW irradiation periods. The mean diameter and the size distributions of AgNPs obtained by measuring the diameter and standard deviation of all particles in the images are shown beside each image.

As seen from Figure 1, the mean diameter of the AgNPs slightly increased and depended on MW irradiation times. In the AgNPs solution prepared at 20 and $40 \mathrm{~s}, \mathrm{AgNPs}$ were formed with a broad size distribution and the mean particle size and standard deviation were about $7.14 \pm 4.31$ and $9.15 \pm 3.71 \mathrm{~nm}$ (Figure 1 A,C), respectively. There is also evidence of small species as well as large ones, as can be seen from Figure $2 \mathrm{E}$ and $\mathrm{G}$. When the irradiation period was increased to 60 and $90 \mathrm{~s}$, the mean particles size of AgNPs was increased considerably to $10.1 \pm 3.31$ and $11.5 \pm 2.59 \mathrm{~nm}$, respectively. This result also indicated that with increased MW-irradiation time, the AgNPs slightly increased in the solvent [14]. However, the result showed a narrow size distribution of the AgNPs, indicating that the particles were highly homogeneous after $90 \mathrm{~s}$, when the MW-induced fragmentation of large AgNPs gradually commenced. On the other hand, there is an apparent increase in concentration and, probably, the amount of different species after irradiation. However, there is a lack of larger AgNPs, whereas many small NPs appeared. The particle size distribution obtained from the TEM image (Figure 1) shows that the combined effect of PVP thus helps in controlling the size of the particles. 
Figure 1. Transmission electron microscopy (TEM) images and the corresponding particle size distributions of Ag nanofluids at different $\mathrm{MW}$-irradiation times: $20 \mathrm{~s}$ (A,B), $40 \mathrm{~s}(\mathbf{C}, \mathbf{D}), 60 \mathrm{~s}(\mathbf{E}, \mathbf{F})$, and $90 \mathrm{~s}(\mathbf{G}, \mathbf{H})$.
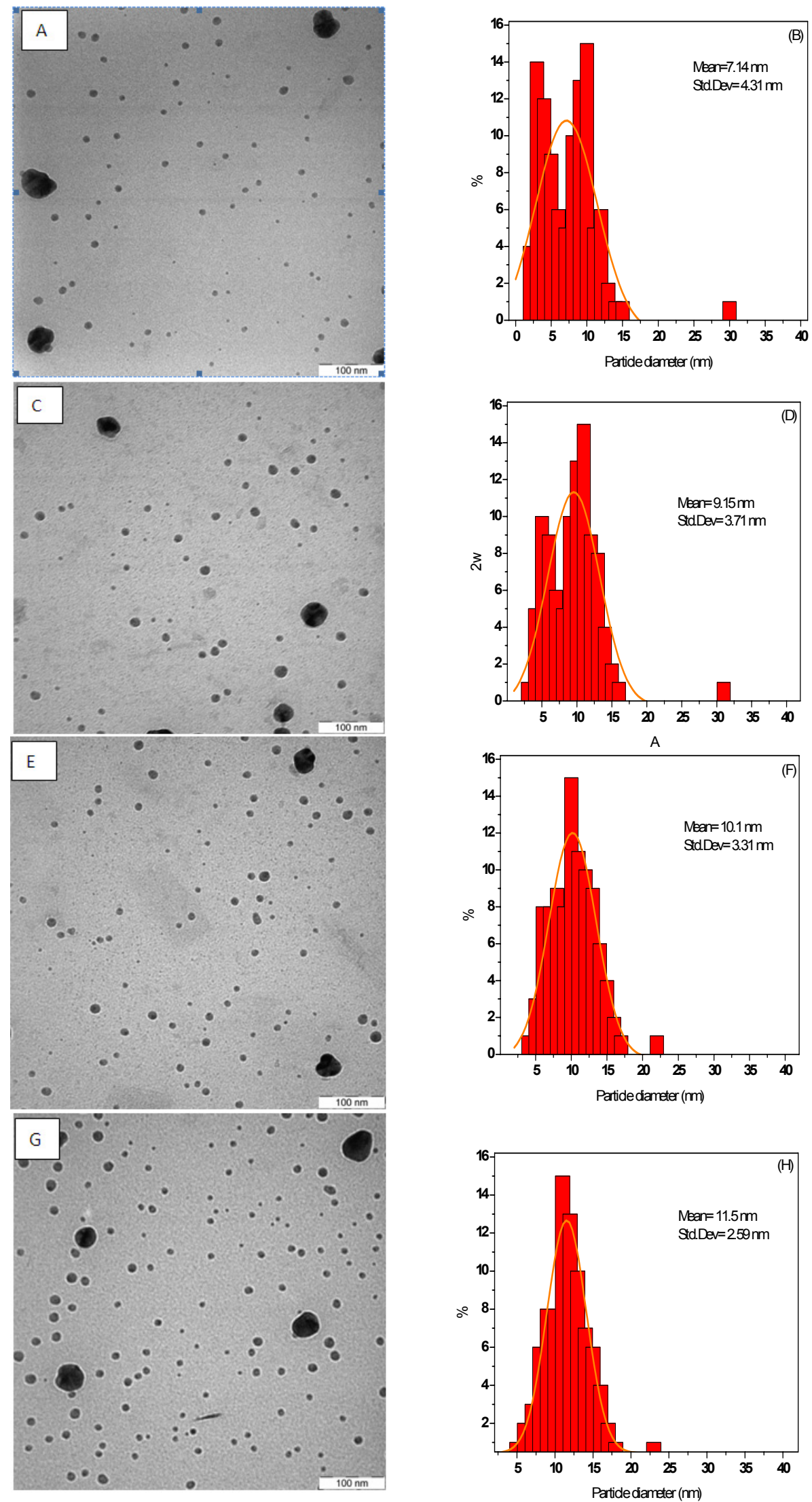
Figure 2. TEM micrographs of the dendritic nanostructures forming Ag trees.

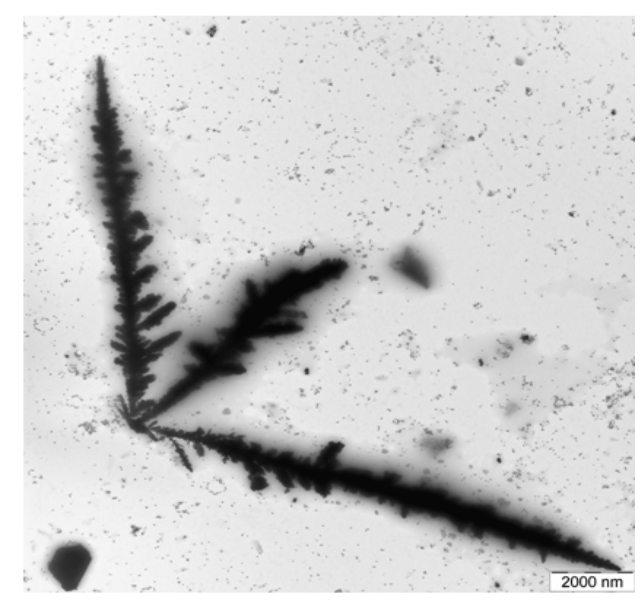

Figure 2 shows a TEM image of AgNPs that was formed at $90 \mathrm{~s}$ of MW irradiation on the solution. As seen from Figure 2, the formation of dendritic nanostructures to the formation of silver trees was observed. When the concentration of the tne AgNPs precursor was increased, large dendritic nanostructures were formed [25]. Dendritic structures of AgNPs are formed at the interface when $\mathrm{Ag}$ ions were reduced in the presence of surfactants. Dendritic nanostructures of $\mathrm{Ag}$ have been produced by MW irradiation of $\mathrm{AgNO}_{3}$ in the presence of PVP [26]. According to the suggestion for the creation of Ag dendrites, MW power and PVP as a surfactant play important roles in the formation of Ag dendrites. Ag ions were reduced and metallic AgNPs were formed at the beginning of the reaction by MW irradiation of $\mathrm{AgNO}_{3}$ in the presence of PVP. Therefore, the interaction between Ag particles and MW energy can lead to the growth of the nanoparticles and an increase in temperature in nearby areas and may encourage new nuclei to appear at its boundary for further diffusion-limited growth. Consequently, the rate of the nucleation and development of the crystal control the creation of the dendrites [10].

\subsubsection{Photon Correlation Spectroscopy}

Figure 3 shows the hydrodynamic density size distribution and the mean diameter of the colloidal AgNPs that were measured by PCS during MW irradiation periods. The mean diameters of AgNPs and the range of AgNPs particles in the solution are summarized in Table 1. The result showed that the maximum particle distribution and a monodispersion of particles were reached after $90 \mathrm{~s}$ of irradiation time, the distribution peaks being around $110.66 \mathrm{~nm}$ and the NPs sizes ranging from 99.14 to $123.65 \mathrm{~nm}$, which was highly homogeneous. The sharper curve of distribution of the particle sizes is equivalent to a higher concentration of particle sizes in the nanofluid. Consequently, the present process can produce AgNPs steadily with almost equivalent quality and yield. This phenomenon was also confirmed in the particle size distribution obtained from the TEM image (Figure 1G). In all nanoparticle cases, the measured particle sizes were 10 times larger than the values obtained by TEM analysis of dried powder. The PCS measurement indicates the effective diameter of the agglomerates which implies that the surface of AgNPs was coated by PVP molecules, since they could not be detected in the solid state by TEM but were interpreted as a part of NPs in solution when recorded by PCS [12]. In the PCS measurement, all suspensions were identically diluted, and the differences in 
particle size and aggregate morphology reported here are valid for relative comparisons between the size distributions. However, the scattered light intensity is inversely proportional to the particle size. When the sample contains particles with a wide size distribution, or particles are mixed with surfactant coated molecules, the signal from larger particles can significantly block the signal from smaller particles, which makes accurate measurement difficult to realize. In this study, the large difference between the TEM images and the PCS data agrees with this assumption. Probably, the average size $(\sim 100 \mathrm{~nm})$ of the PCS data (Figure 3) reflects the small amount of large AgNPs as shown in the TEM images (Figure 1).

Figure 3. The hydrodynamic density size distribution of the colloidal Ag-NPs solutions that was measured by photon correlation spectroscopy (PCS) analyzer at different irradiation time: 20 (W1), 40 (W2), 60 (W3), and $90 \mathrm{~s}$ (W4).

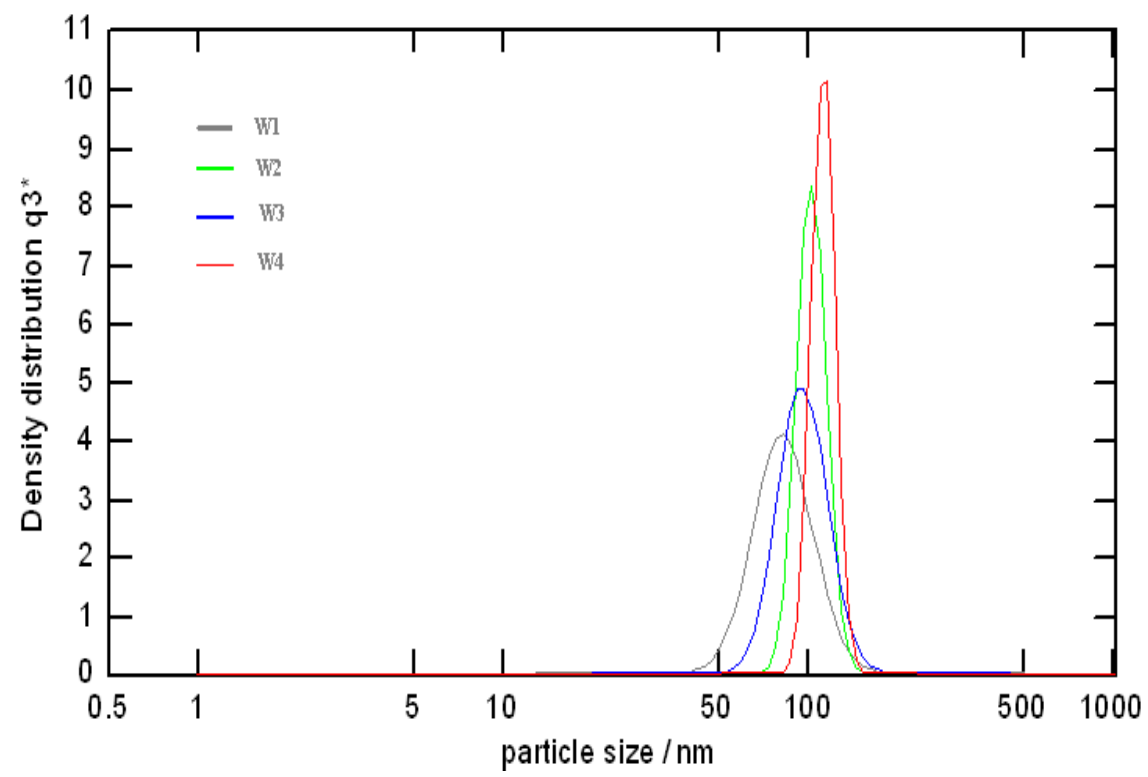

Table 1. The hydrodynamic mean diameter of Ag-NPs solutions prepared by MW irradiation in aqueous solutions at different irradiation times measured by PCS analyzer.

\begin{tabular}{ccc}
\hline MW Irradiation Time (s) & Ag-NPs Mean Diameter $(\mathbf{n m})$ & Standard Deviation $(\mathbf{n m})$ \\
\hline 20 & 81.51 & 23.86 \\
40 & 94.49 & 22.97 \\
60 & 101.25 & 19.45 \\
90 & 110.66 & 12.25 \\
\hline
\end{tabular}

\subsubsection{UV-vis Spectra}

Figure 4(a) shows the absorption spectra of the colloidal AgNPs during different MW irradiation times. The UV-vis spectra showed symmetric peaks at about $420 \mathrm{~nm}$, which is distinctive of surface plasmon resonance (SPR) features of AgNPs colloidal solutions, and a long tail lengthening toward the lower wavelength (UV range) [27]. However, the surface plasmon absorption band obtained from the $\mathrm{AgNO}_{3} /$ water suspension (W0) before the MW-irradiation showed no sign of colloidal silver. Further a wide band absorption at around $450 \mathrm{~nm}$ is shown. The UV-vis spectra of the samples showed that the 
absorbance of AgNPs colloidal solutions increased sharply when the irradiation MW times were increased to 20,40,60, and $90 \mathrm{~s}$, respectively, weherby the SPR peaks that appeared were slightly red-shifted and increased to 419 (W1), 420 (W2), 421(W3), and 421(W4) nm, respectively. The result showed that the maximum absorption and a narrow size distribution of the AgNPs were reached after $90 \mathrm{~s}$ of irradiation time, where it was highly homogeneous and the increase of absorbance was due to the surge of the number of NPs in the solution [28].

For a stability test of AgNPs, the absorption spectra of nanofluids of sample W3 was taken after storage for six months at room temperature (Figure $4 \mathrm{~b}$ ). The fact that the absorption peaks red shifted only slightly from 420 to $427 \mathrm{~nm}$ means that the agglomeration of AgNPs was quite small, and the small change in dispersion intensity shows that the AgNPs solution was quite stable. Therefore, AgNPs produced by MW irradiation produce stable AgNPs over a long period of time.

Figure 4. (a) Evolution of UV spectra during the formation of silver colloids in aqueous solution. Ag nanofluids at different MW irradiation times. The time of irradiation is shown on the traces. (b) The UV-vis spectra of AgNPs prepared using MW irradiation at $60 \mathrm{~s}$ (W3), fresh and after storage for six months at room temperature.

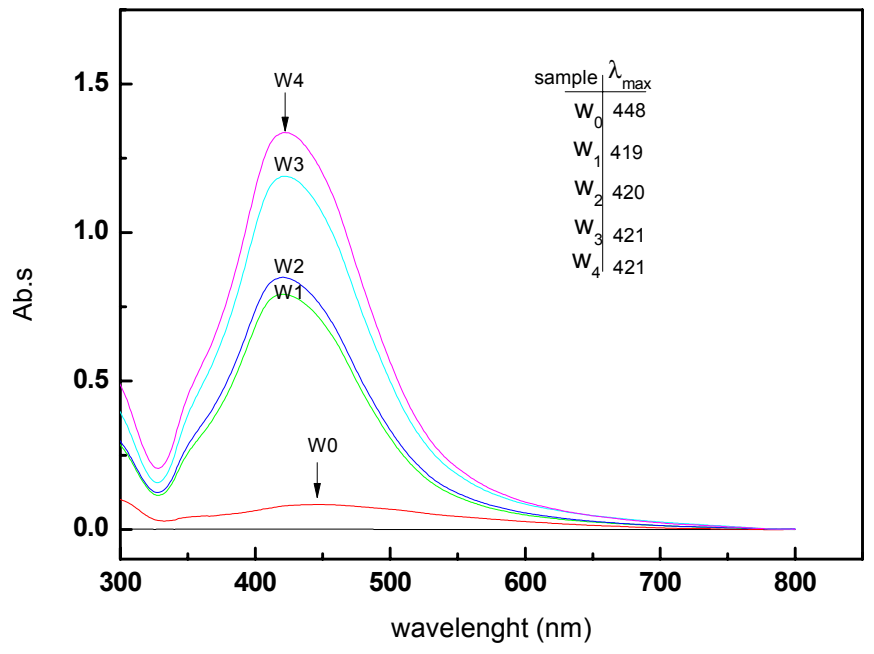

(a)

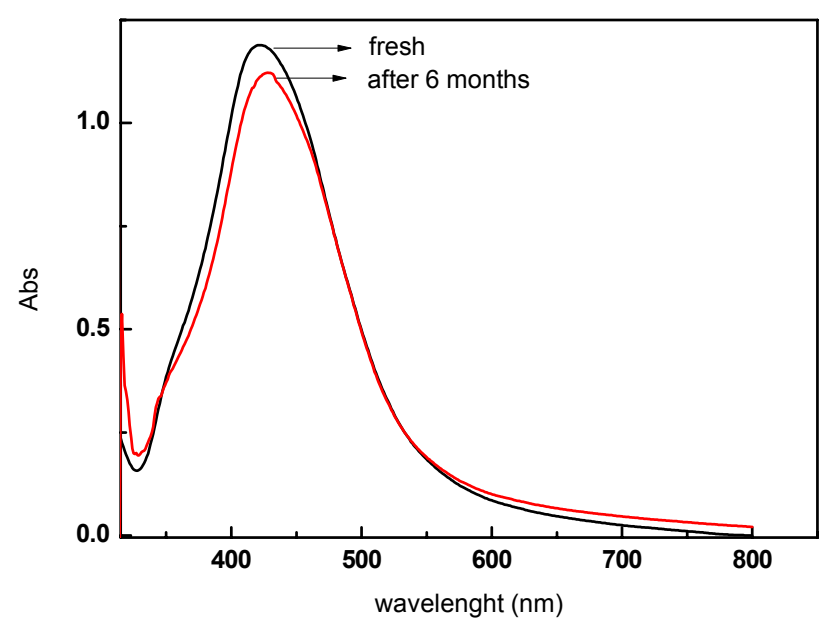

(b)

\subsection{Formation of AgNPs with Conventional Heating}

Figure 5 presents the UV-vis spectra of AgNPs dispersions synthesized using MW irradiation and conventional heating methods. The absorption peak intensity of the dispersion obtained by MW heating was higher and blue shifted (from 435 to $421 \mathrm{~nm}$ ) compared to that of conventional heating. This blue shift and high peak indicate that the size of AgNPs prepared in MW heating is smaller and has a higher density [9] compared with that of conventional heating, which reflects more uniform size distributions. The obtained concentration of AgNPs by MW irradiation and conventional heating technique was 0.136 and $0.102 \mathrm{mg} / \mathrm{L}$, respectively, confirming a high density of AgNPs produced by the MW method which confirmed the observation in the UV-vis spectra (Figure 5).

In Figure 6, the TEM image and the histogram clearly show that the AgNPs obtained from the conventional heating are bigger, $14.5 \mathrm{~nm}$, than those produced with the MW heating method and not uniform in size, based on big standard deviation of $7.73 \mathrm{~nm}$. The decrement in size of AgNPs produced 
by MW irradiation can be attributed to the efficiency of the electromagnetic field in heating the polarizing water molecules. With conventional heating, the solvent is heated by conduction and convection, so that there is a large temperature distribution within the solvent [10]. Rapid heating by MW irradiation produced a narrow size distribution of AgNPs because of homogeneous nucleation and subsequent crystal growth which was indicated by a superior yield of AgNPs [14].

Figure 5. The UV-vis spectra of Ag-NPs prepared with MW irradiation and thermal method at the same temperature, the concentrations of silver nitrate and PVP are the same, while the temperatures reach $60^{\circ} \mathrm{C}$.

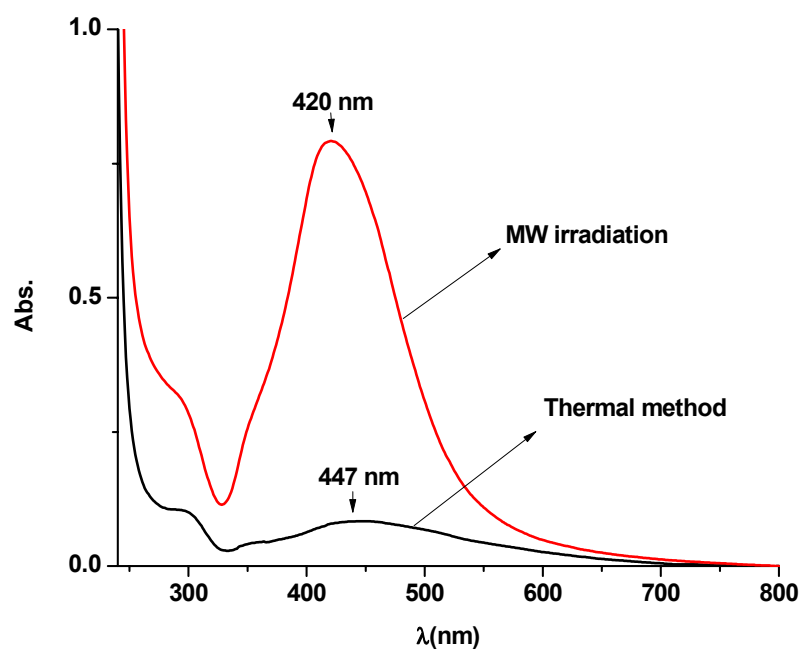

Figure 6. The TEM images of Ag-NPs synthesized by conventional heating method.
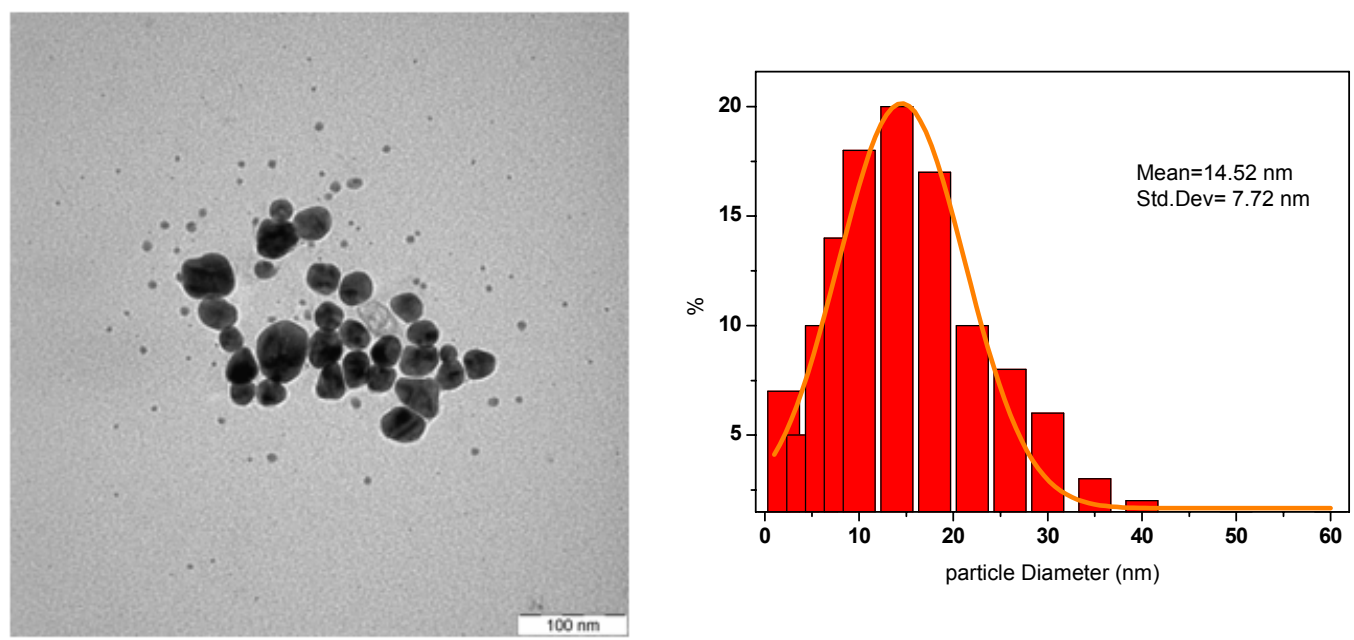

\section{Conclusions}

The preparation of AgNPs in water and in presence of a PVP solution was done by MW irradiation as a simpler and greener method than heating by conventional method. The fabrication of narrowly dispersed silver nanoparticles was tuned in the size range of 7-17 $\mathrm{nm}$ in diameter with a standard deviation of $\sigma \leq 4.3-2.5 \mathrm{~nm}$, by varying irradiation times only from 20 to $90 \mathrm{~s}$. This showed that they were very stable in aqueous solution without any sign of precipitants over the long period of six months. The results indicated that by using MW irradiation, it is possible to obtain AgNPs of regular 
shape, narrow size distribution and a higher degree of crystallization in a reasonable synthesis time. The fast MW heating produces smaller and more uniform particle sizes and more density compared to that of the conventional heating method. Additionally, we believe that determination of the hydrodynamic diameter of the colloidal AgNPs under the PCS analyzer may be very useful for further nanomedicine applications to monitor their agglomeration state. Such highly-monodispersed and size-controlled AgNPs will contribute to the size-dependent nanomedicine applications of AgNPs.

\section{Acknowledgement}

The authors are grateful to the Ministry of Higher Education of Malaysia for supporting this work under Fundamental Research Grant Scheme (FRGS) of No.01-04-10-864FR.

\section{References}

1. Sclafani, A.; Herrmann, J. Influence of metallic silver and of platinum silver bimetallic deposits on the photocatalytic activity of titania (anatase and rutile) in organic and aqueous media. J. Photochem. Photobiol. A 1998, 113, 181-188.

2. Jiang, H.; Moon, K.; Zhang, Z. Variable frequency microwave synthesis of silver nanoparticles. J. Nanopart. Res. 2006, 8, 117-124.

3. Sun, Y.G.; Mayers, B.T.; Herricks, Y. Crystalline silver nanowires by soft solution processing. Nano Lett. 2003, 3, 955-960.

4. Darroudi, M.; Ahmad, M.B. Preparation and characterization of gelatin mediated silver nanoparticles by laser ablation. J. Alloy Compd. 2011, 509, 1301-1304.

5. Carotenuto, G.; Pepe, G. Preparation and characterization of nano-sized Ag/PVP composites for optical applications. Eur. Phys. J. B 2000, 16, 11-17.

6. Clary D.R.; Mills, G. Preparation and thermal properties of CuO particles. J. Phys. Chem. C 2011, 115, 14656-14663.

7. Bilecka, I.; Elser, P.; Niederberger, M. Kinetic and thermodynamic aspects in the microwave-assisted synthesis of ZnO nanoparticles in benzyl alcohol. ACS Nano 2009, 3, 467-477.

8. Zhu, J.F.; Zhu, Y.J.; Ma, M.G. Simultaneous and rapid microwave synthesis of polyacrylamide-metal sulfide $\left(\mathrm{Ag}_{2} \mathrm{~S}, \mathrm{Cu}_{2} \mathrm{~S}, \mathrm{HgS}\right)$ nanocomposites. J. Phys. Chem. C 2007, 111, 3920-3926.

9. Mohamed, M.B.; AbouZeid, K.M.; Abdelsayed, V.A. Growth mechanism of anisotropic gold nanoparticles via microwave synthesis: formation of dioleamide by gold nanocatalysis. ACS Nano 2010, 4, 2766-2772.

10. Tsuji, M.; Hashimoto, M.; Nishizawa, Y. Microwave rapid heating has received considerable attention as a new promising method for the one-pot synthesis of metallic nanostructures in solutions. Chem. Eur. J. 2005, 11, 440-452.

11. Sun, Y.; Mayers, B.; Herricks, T. Polyol synthesis of uniform silver nanowires: a plausible growth mechanism and the supporting evidence. Nano Lett. 2003, 3, 955-960.

12. Wada, Y.; Kuramoto, H. Microwave-assisted size control of CdS nanocrystallites. J. Mater. Chem. 2001, 11, 1936-1940.

13. Kundu, S.; Peng, L. New route to obtain high-yield multiple-shaped gold nanoparticles in aqueous solution using microwave irradiation. Inorg. Chem. 2008, 47, 6344-6352. 
14. Acharya, S.; Singh, K. Microwave-assisted chemical reduction routes for direct synthesis of (fct) L10 phase of Fe-Pt. J. Microw. Power Electromagn. Energy 2011, 45, 63-69.

15. He, R.; Qian, X.; Yin, J. Formation of silver dendrites under microwave irradiation. Chem. Phys. Lett. 2003, 369, 454-458.

16. Zhou, X.; Huang, X.; Qi, X. In situ synthesis of metal nanoparticles on single-layer graphene oxide and reduced graphene oxide surfaces. J. Phys. Chem. C 2009, 113, 10842-10846.

17. Hood, E. Nanotechnology: Looking as we leap. Environ. Health Perspect. 2004, 112, 740-749.

18. Acharya, S.A.; Khule, S.M.; Singh, K.; Bhoga, S.S. Direct Synthesis of Fe-Pt Nanoparticles in Ordered Face Centered (fct) $\mathrm{LI}_{\mathrm{o}}$ Phase by Microwave Assisted Route. In Proceedings of the 55th DAE Solid State Physics Symposium, Manipal, India, 26-30 December 2010; pp. 371-372.

19. Hsiao, M.; Lin, C.; Chang, Y.; Chen, L. Ultrasonic mixing and closed microwave irradiation-assisted transesterification of soybean oil. Fuel 2010, 89, 3618-3622.

20. Chen, G.; Zhu, F.; Sun, X.; Sun, S.; Chen, R. Benign synthesis of ceria hollow nanocrystals by a template-free method. CrystEngComm 2011, 13, 2904-2908.

21. Chung, S.J.; Leonard, J.P. Characterization of $\mathrm{ZnO}$ nanoparticle suspension in water: Effectiveness of ultrasonic dispersion. Powder Technol. 2009, 194, 75-80.

22. McConnell, D.R. Energy consumption: A comparison between the microwave oven and the conventional electric rang. J. Microw. Power 1974, 9, 341-347.

23. Cheng, W.M.; Raghavan, G.S.V.; Ngadi, M.; Wang, N. Microwave power control strategies on the drying process. I. Development and evaluation of new microwave drying system. J. Food Eng. 2006, 76, 188-194.

24. Link, S.; El-Sayed, M.A. Size and temperature dependence of the plasmon absorption of colloidal gold nanoparticles. J. Phys. Chem. B 1999, 103, 4212-4217.

25. Baruwati, B.; Polshettiwar V. Glutathione promoted expeditious green synthesis of silver nanoparticles in water using microwaves. Green Chem. 2009, 11, 926-930.

26. Agrawal, V.V.; Kulkarni, G.U. Surfactant-promoted formation of fractal and dendritic nanostructures of gold and silver at the organic-aqueous interface. J. Colloid Interface Sci. 2008, 318, 501-506.

27. Tsuji, T.; Iryo, K. Preparation of silver nanoparticles by laser ablation in solution: Influence of laser wavelength on particle size. Appl. Surf. Sci. 2002, 202, 80-85.

28. Kappe, C.O. Controlled microwave heating in modern organic synthesis. Angew. Chem. Int. Ed. 2004, 43, 6250-6284.

(C) 2012 by the authors; licensee MDPI, Basel, Switzerland. This article is an open access article distributed under the terms and conditions of the Creative Commons Attribution license (http://creativecommons.org/licenses/by/3.0/). 